Research Paper

\title{
Angiotensin 1-7 Overexpression Mediated by a Capsid- optimized AAV8 Vector Leads to Significant Growth Inhibition of Hepatocellular Carcinoma In vivo
}

Yingying Mao ${ }^{*}$, Nana Pei2 ${ }^{*}$, Xinglu Chen ${ }^{3 *}$, Huiying Chen ${ }^{1}$, Renhe Yan ${ }^{4}$, Na Bai ${ }^{5}$, Andrew Li' ${ }^{6}$, Jinlong Li1, Yanling Zhang ${ }^{1}$, Hongyan Du ${ }^{1}$, Baihong $\mathrm{Chen}^{1}{ }^{1}$, Colin Sumners ${ }^{7}$, Xuejun Wang ${ }^{8 凶}$, Shengqi Wang ${ }^{8 凶}$, Hongwei $\mathrm{Li}^{1 凶}$

1. School of Laboratory Medicine and Biotechnology, Southern Medical University, Guangzhou, Guangdong, China;

2. Department of Clinical Pathology, The First Affiliated Hospital of Jinan University, Guangzhou, Guangdong, China;

3. Clinical Laboratory, The First Affiliated Hospital of Guangdong Pharmaceutical University, Guangzhou, Guangdong, China;

4. Guangzhou Bioneeds Biotechnology CO., LTD, Guangzhou, Guangdong, China;

5. Deparement of Nuclear Medicine, People's Hospital of Yuxi City, Yuxi, Yunnan, China.

6. Department of Biomedical Engineering, The Johns University School of Medicine, Baltimore, USA;

7. Departments of Physiology and Functional Genomics, University of Florida, Gainesville, Florida, USA.

8. Department of Biotechnology, Beijing Institute of Radiation Medicine, Beijing, China.

*These authors contributed equally to this work

$\triangle$ Corresponding authors: hongwei1@yahoo.com; sqwang@bmi.ac.cn; xjwang@bmi.ac.cn

(C) Ivyspring International Publisher. This is an open access article distributed under the terms of the Creative Commons Attribution (CC BY-NC) license (https://creativecommons.org/licenses/by-nc/4.0/). See http://ivyspring.com/terms for full terms and conditions.

Received: 2017.08.04; Accepted: 2017.12.14; Published: 2018.01.11

\begin{abstract}
Background: Angiotensin-(1-7) [Ang-(1-7)] has been identified to inhibit the growth of many types of tumor cells both in vitro and in vivo. However, the rapid degradation of Ang-(1-7) in vivo limits its clinical application. Adeno-associated virus (AAV) serotype-8 is a remarkable vector for long-term in vivo gene delivery.

Method: This study was designed to investigate the effects of AAV-mediated Ang-(1-7) overexpression on hepatocellular carcinoma. We first generated three different tyrosine $(Y)$ to phenylalanine (F) mutants of AAV8 (Y447F, Y703F, Y708F) and evaluated their in vivo transduction efficiencies.

Results: The data indicated that the Y703F mutant elicited a significant enhancement of liver gene delivery when compared with wild-type AAV8 (wtAAV8). The anti-tumor effect of Ang-(1-7) mediated by this optimized vector was evaluated in $\mathrm{H} 22$ hepatoma-bearing mice. Our results demonstrated that AAV-Ang-(1-7) persistently inhibited the growth of hepatocellular carcinoma by significantly downregulating angiogenesis. This was confirmed by observed decreases in the levels of the proangiogenic factors VEGF and PIGF.

Conclusion: Collectively, these data suggest that Ang-(1-7) overexpression mediated by the optimized vector may be an effective alternative for hepatocellular carcinoma therapy due to its long-term and significant anti-tumor activity.
\end{abstract}

Key words: angiotensin-(1-7), hepatocellular carcinoma, adeno-associated virus, gene therapy

\section{Background}

Hepatocellular carcinoma (HCC) is one of the most common and deadly cancers worldwide and the second leading cause of cancer-related mortality in China $[1,2]$. Currently, surgery supplemented with chemotherapeutic agents is the main HCC therapeutic schedule. However, the low sensitivity, severe adverse effects and an unprecedented high cost has restricted the use of chemotherapeutic agents for 
patients with HCC [3]. Moreover, the high rate of metabolism and the existence of multidrug resistant transporter proteins in tumor cells further diminish the efficacy of current therapeutic regimens for HCC [4]. Therefore, the development of more effective alternative strategies for the treatment of HCC is a matter of great urgency.

The renin-angiotensin system (RAS) has been identified to play an important role in the pathogenesis of cancers, including prostate cancer, lung cancer, nasopharyngeal carcinoma and others [5-7]. Angiotensin-(1-7) [Ang-(1-7)], an endogenous heptapeptide hormone of the RAS, mediates both vasodilation and anti-proliferative effects by activating a distinct plasma membrane G proteincoupled receptor identified as the Mas receptor (MasR) $[8,9]$. There is a growing body of evidence suggesting that Ang-(1-7) exerts anti-tumor effects in a variety of cancers, mainly through its anti-proliferative and anti-angiogenic actions [6, 7]. In fact, a phase I/II clinical trial has confirmed the effectiveness and safety of Ang-(1-7) in patients with advanced solid tumors [10-12]. Ang-(1-7) has been regarded as a promising anti-tumor therapeutic drug despite its half-life being very short ( $\sim 30 \mathrm{~min}$ in humans and 20s in rodents), which severely hampers the in vivo efficacy of this peptide [13].

Adeno-associated virus (AAV) is one of the most promising vectors for human gene therapy due to its persistence, high efficiency and lack of pathogenicity in gene delivery. It is noteworthy that the European Medicines Agency approved an AAV1 mediated LPL (S447X) gene therapeutic agent for Lipoprotein lipase-deficiency in 2012 [14]. Clinical trials have confirmed long-term efficacy and safety of AAV mediated gene therapy in patients with leber congenital amaurosis type 2 or hemophilia B [15, 16]. Moreover, AAV has achieved favorable effects in liver, retina, brain, cardiovascular system and muscle gene therapy with long-term and non-toxic transgene expression [17-19]. AAV8 has been identified as a remarkable gene delivery vector in vivo [20]. It has been reported that a single tyrosine mutation on the capsid of AAV8 allows vectors to evade intracellular phosphorylation and subsequent ubiquitination and proteasome-mediated degradation, resulting in dramatic enhancement of gene expression [21, 22].

The present study was designed to explore the anti-tumor effect of Ang-(1-7) in H22 hepatoma-bearing mice mediated by an optimized AAV8 vector. We constructed three tyrosine mutants of AAV8: Y447F, Y703F and Y708F. These capsid mutants and the vectors containing dual-luciferase gene were packaged and their in vivo transduction efficiency was evaluated in C57BL/ 6 mice. Then the optimized mutant was used to mediate long-term and stable expression of Ang-(1-7) in a murine hepatocellular carcinoma model. Our results showed that overexpression of Ang-(1-7) significantly inhibits tumor growth by decreasing angiogenesis and cell proliferation.

\section{Materials and Methods}

\section{Cell culture}

Human embryonic kidney cell line HEK-293T, murine hepatocellular carcinoma cell line H22, human hepatocellular carcinoma cell lines QGY-7701, Bel-7402, SMMC-7721, HepG2 and normal human liver cell line $\mathrm{LO} 2$ were all obtained from the American Type Culture Collection (ATCC, Manassas, VA). Cells were maintained as monolayer cultures in Dulbecco's modified Eagle's medium (DMEM) (Hyclone, Logan, UT). Media were supplemented with 10\% fetal bovine serum (FBS) (Hyclone), 100units/ml penicillin, $100 \mathrm{mg} / \mathrm{ml}$ streptomycin (Invitrogen, Carlsbad, CA, USA) and maintained in $5 \% \mathrm{CO}_{2}$ at $37^{\circ} \mathrm{C}$.

\section{Generation of AAV8 mutants and recombinant vectors}

Phosphorylation and ubiquitination sites on the AAV8 capsid were determined using online prediction tools $[23,24] . \mathrm{Y} \rightarrow \mathrm{F}$ mutations: $\mathrm{Y} 447 \mathrm{~F}$, Y703F, Y708F, were introduced in the AAV8 rep/cap plasmids by overlapping PCR. The primers used are shown in Table 1. The presence of the desired point mutation was verified by DNA sequencing. Recombinant AAV vectors containing dual-luciferase reporter or Ang-(1-7) were generated by polyethylenemine (PEI)-based triple transfection of 293 T cells and were purified by polyethylene glycol (PEG) precipitation followed by double $\mathrm{CsCl}$ gradient purification [25]. After three changes of dialysis in virus dialysis buffer ( $1 \mathrm{x}$ phosphate-buffered saline [PBS], 2\% mannitol, $6 \mathrm{mM} \mathrm{MgCl}_{2}$ ) at room temperature for 6 to 8 hours or at $4^{\circ} \mathrm{C}$ overnight, vector genome copy titers were determined by quantitative PCR.

\section{Evaluation of in vivo gene transduction efficiency}

Male C57BL/ 6 mice of 6-8 weeks were obtained and housed in the animal center of the Academy of Military Medicine Science. All animals received humane care and the study protocol complied with the institution's ethics guidelines. To evaluate the transduction effect in vivo, animals were injected with $1 \times 10^{10}$ vector genomes (vg) for each of AAV-Gluc$2 \mathrm{~A}$-Fluc in equal volumes via the tail vein. For each 
group, 6-8 mice were used. All animals received humane care and the study protocol complied with the institution's ethics guidelines.

Table 1. Primer sequences of the three single point mutations on the AAV8 capsid

\begin{tabular}{lll}
\hline Residue & Sequence (5 ${ }^{`}$ to ${ }^{`}$ ) & Nucleotide change \\
\hline Y447F & Wild-type primer sequence: & TAC $\rightarrow$ TTC \\
& TACCTGTACTACTTGTCT & \\
& Mutant primer sequence: & \\
& TACCTGTACTTCTTGTCT & \\
Y703F & Wild-type primer sequence: & TAC $\rightarrow$ TTC \\
& ATCCAGTACACCTCCAAC & \\
& Mutant primer sequence: & \\
ATCCAGTTCACCTCCAAC & TAC $\rightarrow$ TTC \\
& Wild-type primer sequence: & \\
& AACTACTACAAATCTACA & \\
& Mutant primer sequence: & \\
& AACTACTTCAAATCTACA & \\
\hline
\end{tabular}

\section{Luciferase activity assay}

At 7, 14, 21 and 28 days post vector administration, blood was collected via the tail vein. Secretory Gaussia princeps luciferase (Gluc) activity was measured using BioLux ${ }^{\circledR}$ Gaussia Luciferase Flex Assay Kit (New England Biolabs) at different time points.

\section{In vivo animal Bioluminescence Image Analysis}

At day 42 after vector injection, mice were anaesthetized with pentobarbital sodium (1\%, $40 \mathrm{mg} / \mathrm{kg}$ mice) via intraperitoneal injection. The D-luciferin substrate (Biotium, Hayward, CA) was injected intraperitoneally at a dose of $150 \mu \mathrm{g} / \mathrm{g}$. The mice were then placed in a light-tight chamber, and images were generated using a Bioanalytical Instruments (Berthold Technologies, DE). Light was monitored in all of the experiments described at 8-12 minutes after the substrate injection. The visual output represents the number of photons emitted/second $/ \mathrm{cm}^{2}$ as a false color image where the maximum is red and the minimum is dark blue.

\section{Genome copy determination}

After bioluminescence imaging, mice were killed. Different tissues were collected from each mouse administered with either wild-type AAV8 or the mutant vectors. Genomic DNA was isolated using the TIANamp Genomic DNAKit (TIANGEN BIOTECH CO., LTD, Beijing, China) according to the manufacturer's protocol. Total DNA concentration was determined by spectrophotometry using a Nanodrop. Quantitative PCR was used to determine the vector copy numbers in $100 \mathrm{ng}$ of template genomic DNA by amplifying the firefly luciferase sequence (forward primer: ACTGCCTGCGTGAGATTCTC; reverse primer: CAGAGTGCTTTTGGCGAAGA).
Glyceraldehyde-3-phosphate dehydrogenase (GAPDH) was used as the housekeeping control gene. Data were captured and analyzed using ABI Prism 7500 sequence detection system version 1.1 software (Life Technologies).

\section{Comparison of the in vivo transduction efficiency between AAV-8 wild type and its capsid mutant AAV8-Y703F using Ang-(1-7) as a target gene}

Male BALB/c mice aged 4 to 5 weeks were purchased from the Institute of Comparative Medicine and Center of Laboratory Animals of the Southern Medical University (SMU). Animal handling and experimental procedures were approved by the Animal Experimental Ethics Committee of SMU. Mice were injected with $1 \times 10^{11}$ vector genomes $(\mathrm{vg})$ each of recombinant AAV8-Ang-(1-7) or Y703F-Ang-(1-7) in equal volumes via the tail vein. At $7,14,21$ and 28-days post vector administration, serum Ang-(1-7) was detected by ELISA (Bachem). Further, liver genomic DNA was isolated using the TIANamp Genomic DNA Kit (TIANGEN BIOTECH CO., LTD, Beijing, China) according to the manufacturer's protocol. Total DNA concentration was determined by spectrophotometry using a Nanodrop. Quantitative PCR was used to determine the vector copy numbers in $100 \mathrm{ng}$ of template genomic DNA by amplifying the Ang-(1-7) fusion protein sequence (forward primer: GACGGTTCTTACTTCATAT; reverse primer: ATGGTCTTCTTCAGG). Glyceraldehyde-3-phosphate dehydrogenase (GAPDH) was used as the housekeeping control gene. In addition, liver toxicity of vectors was evaluated by hematoxylin-eosin staining at the end of the experiment.

\section{Anti-tumor growth assay}

Male BALB/c mice aged 4 to 5 weeks were purchased from the Institute of Comparative Medicine and Center of Laboratory Animals of the Southern Medical University (SMU). Animal handling and experimental procedures were approved by the Animal Experimental Ethics Committee of SMU. BALB/c mice were injected with $1 \times 10^{7} \mathrm{H} 22$ cells in $0.1 \mathrm{~mL}$ phosphate buffered saline (PBS) into the right upper back to establish the hepatoma model. When the tumors reached $\sim 100 \mathrm{~mm}^{3}$, the mice were randomly assigned to three groups and received tail vein injections of Y703F-Ang-(1-7) $\left(1 \times 10^{11} \mathrm{vg} /\right.$ mice $)$, Y703F-Luc $\left(1 \times 10^{11} \mathrm{vg} /\right.$ mice $)$ or PBS. Each group contained 6-8 mice and the experiment was repeated 3 times. Tumor size was measured every 3-5 days. The mice were anesthetized on day 21 and euthanized by decapitation and tumors were dissected. Tumor 
volumes were calculated as follows: volume $=(\mathrm{D} \times$ $\left.d^{2}\right) / 2$, where $D$ is the longest diameter and $d$ is the shortest diameter. Sera were also collected for quantification of Ang-(1-7) by ELISA (Bachem).

\section{Immunohistochemical staining}

Both livers and tumors were fixed in 10\% buffered formalin for 24 hours and incubated in $70 \%$ ethanol for 48 hours before embedding in paraffin. The embedded livers and tumors were cut into 5-mm thick sections. Liver sections were stained with H\&E to determine morphology and mouse IgG to indirectly determine Ang-(1-7) overexpression. Cell proliferation in the transplanted tumors was determined with Ki67 (1:200; Abcam) and Cdc6 (1:300; Abcam) antibodies. Angiogenesis was determined by immunostaining with antibodies to VEGF (1:100; Wanleibio, China) and CD34 (1:200; Abcam). The intensity of staining was scored by applying a semiquantitative system, ranging from negative to strong as follows: 0 (negative), 1 (weak), 2 (moderate), and 3 (strong). The percentage of positive cells was categorized according to the positive tumor cells: < $25 \%$ is $1 ; 25 \%$ to $50 \%$ is $2 ; 51 \%$ to $75 \%$ is 3 ; and $>75 \%$ is 4.The histochemical score ( $H$-score) was calculated by multiplying the intensity by the percentage of positive cells [26].

\section{RNA isolation, reverse transcription, and quantitative real-time RT-PCR}

Total RNA in hepatoma cell lines and tumor samples were extracted and subjected to real-time RT-PCR, with the primers used shown as follow:

- h-MasR F: TCACCCACCTGTCTATCGCA R: CACTAATGGCCGTCAGCAGAT

- m-MasR F: AGAAATCCCTTCACGGTCTACA R: TCACCGATAATGTCACGATTGTG

- m-VEGF F: TCACCAAAGCCAGCACATAG R: AATGCTTTCTCCGCTCTGAA

- m-PIGF F: GTGGTGCCTTTCAACGAAGT R: GGACACAGGACGGACTGAAT

- m-Flt-1 F: AGGGATAACAGGCAATTCTGC R: GTGCATCTCTATGAAAGGACTCC

- m-Flk-1 F: TTTGGCAAATACAACCCTTCAGA R: GCTCCAGTATCATTTCCAACCA

- h-GAPDH F: ACGGATTTGGTCGTATTGGG R: CGCTCCTGGAAGATGGTGAT

- m-GAPDH F: TGGCCTTCCGTGTTCCTAC R: GAGTTGCTGTTGAAGTCGCA

The samples were quantified by the comparative $\Delta \Delta \mathrm{Ct}$ method by using human or mouse GAPDH as the internal standard.

\section{Statistical analysis}

The data presented here were expressed as mean \pm standard deviation (SD) and statistical significance was determined by one-way ANOVA followed by Dunnetts post hoc test (compare all columns vs. control group). A $p$-value of $<0.05$ was considered statistically significant. $\mathrm{P}$-values are indicated by asterisks $\left({ }^{* * *} p<0.001,{ }^{* *} p<0.01,{ }^{*} p<0.05\right)$.

\section{Results}

\section{A single tyrosine mutation at the 703 position of the AAV8 capsid leads to a significant enhancement of in vivo gene delivery.}

Phosphorylation and ubiquitination sites on the AAV8 capsid were determined using online prediction tools. The Y447, Y703 and Y708 tyrosine residues might be effective phosphorylation sites, and the three mutants had comparable packaging efficiencies when compared to AAV8 (Table 2), which suggested that the three mutants were compatible modifications on the AAV8 capsid. To explore the transduction efficiency between the three mutants and wild-type AAV8 (wtAAV8), vectors carrying dual-luciferase reporter $\left(1 \times 10^{10} \mathrm{vg}\right)$ were injected into 6-8 week old male C57BL/ 6 mice, via the tail vein. The relative light unit (RLU) emitted by the Gaussia luciferase in blood samples was detected at different time points after vector injection, as shown in Figure 1A. The Gluc expression level in each animal reached a peak at day 21 , and was maintained at a level of 10,000 - 100,000 RLU. Statistical analysis showed that the Y703F mutant elicited significant greater gene expression than wtAAV8, with about 3-fold higher levels at days 7, 14, 21 and 28 after injection, respectively. The Y447F and Y708F mutants showed minimal or no significant difference in ability to increase gene expression when compared to wtAAV8 (Figure 1A). In vivo bioluminescence was analyzed at 42 days post-vector injection. From the ventral view, it can be seen that all vectors display mainly liver tropism, similar to wtAAV8 (Figure 1B). Statistical analyses revealed that the Y703F mutant elicited an enhancement in liver luminescence level of 1.8-fold compared to wtAAV8 (Figure 1C).

Table 2. The average titers of wild-type (wt) AAV8 and its three single point mutant vectors for packaging dual-luciferase gene

\begin{tabular}{ll}
\hline Vector & Physical Titer (Viral Genomes/ml) \\
\hline wtAAV8 & $2.4 \times 10^{12} \mathrm{vg} / \mathrm{ml}$ \\
Y447F & $3.5 \times 10^{12} \mathrm{vg} / \mathrm{ml}$ \\
Y703F & $2.6 \times 10^{12} \mathrm{vg} / \mathrm{ml}$ \\
Y708F & $4.7 \times 10^{12} \mathrm{vg} / \mathrm{ml}$ \\
\hline
\end{tabular}




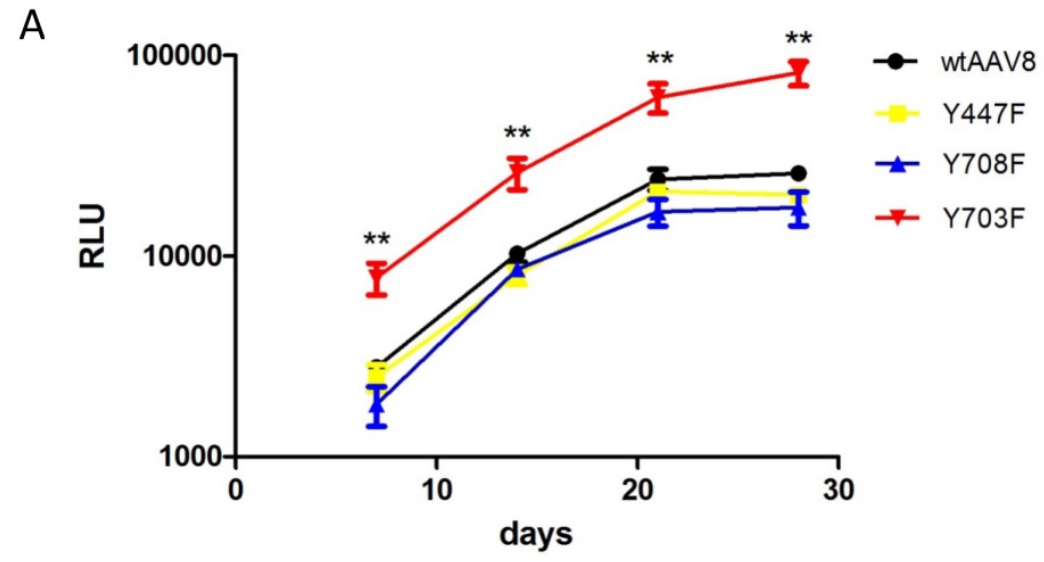

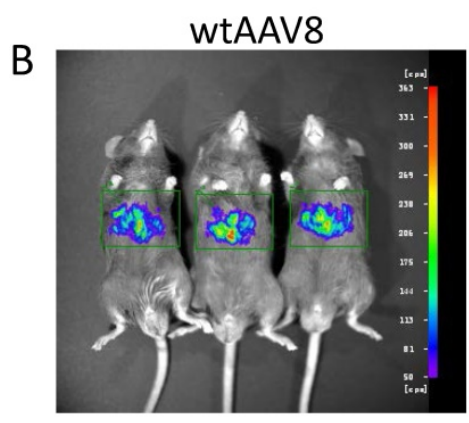
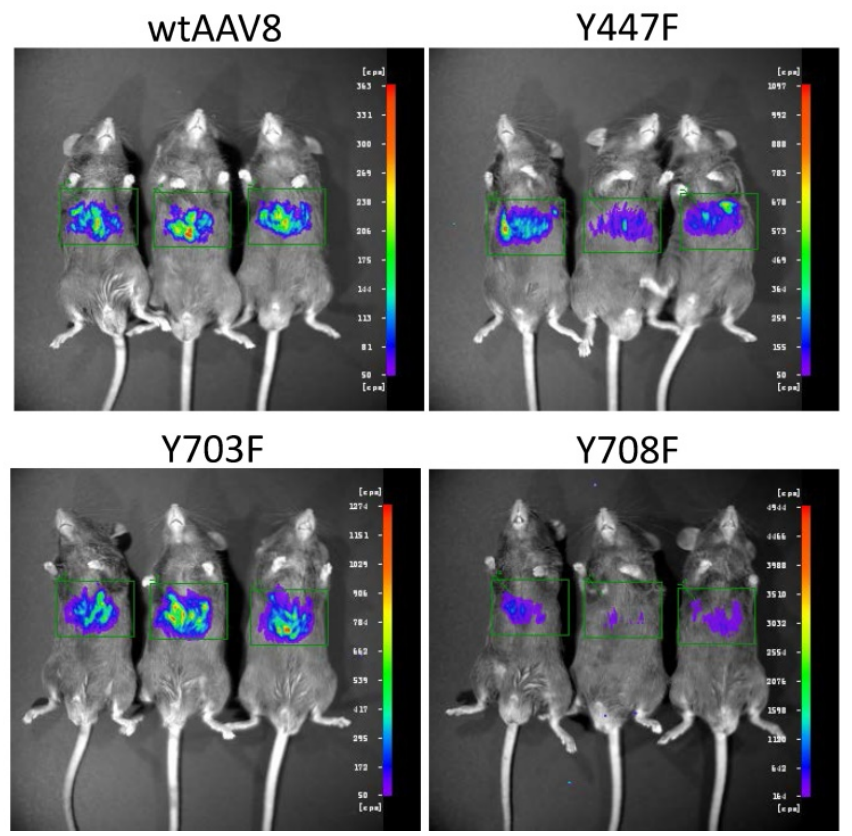

C

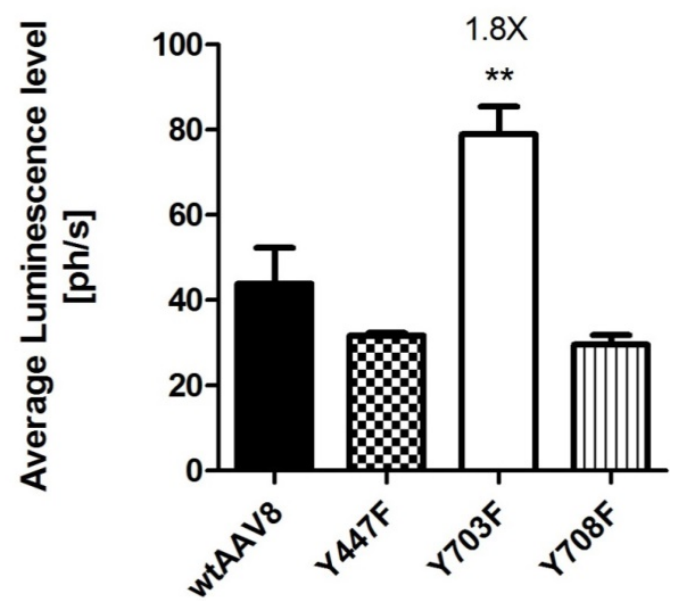

Figure 1. Luciferase expression in C57BL/6 mice after tail-vein injection Gaussia luciferase (Gluc) expression levels were detected at 7, 14,21 and 28 days after vector injection. The Y703F vector elicited sustained and significant enhancement of Gluc from day 7 , with levels that were approximately 3 -fold higher than those in the wtAAV8. Others showed minimal or no significant difference during the experiment (A). For the in vivo bioluminescence analysis, images were taken from the ventral aspect at 6 weeks after vector infusions. The three mutants displayed mainly liver tropism, similar to the wild-type AAV8 (B). Statistical analysis revealed that the Y703F mutant lead to a significant enhancement in liver luminescence levels, 1.8-fold higher than obtained with the wild-type AAV8 (C). The data are shown as mean values $\pm S D,{ }^{*} p<0.05$ compared with wild-type AAV8 groups.

\section{The Y703F mutant of AAV8 elicits significant enhancement of gene transduction in different tissues.}

For further evaluation, we compared the viral genome copy numbers in different tissue samples using real-time PCR. As shown in Figure 2, the Fluc DNA copy number of the four vectors maintained a level of $1 \times 10^{6}-1.5 \times 10^{7}$ GC (genome copies)/100ng DNA in liver tissues. In kidney, heart and gastrocnemius tissues, the numbers dropped to $1 \times 10^{5}$ $1 \times 10^{6}$ GC (genome copies)/100ng DNA, which further illustrates that the four vectors display mainly liver tropism. The mutant Y703F elicited a significant enhancement of gene transduction in liver, kidney, and gastrocnemius tissues with respective 3, 3 and 7-fold higher GC/100ng DNA than wtAAV8, respectively (Figure $2 \mathrm{~A}, 2 \mathrm{~B}, 2 \mathrm{C}$ ). In heart tissue, the $\mathrm{Y} 708 \mathrm{~F}$ mutant resulted in 4-fold higher gene expression when compared with wild-type AAV8 (Figure 2D).

The Y703F mutant of AAV8 elicits a significant enhancement of gene transduction to liver tissue in Balb/c mice.

The different genetic backgrounds of mice may occasionally lead to different outcomes of AAV gene transfer. Thus, we evaluated the transduction efficacy of the Y703F mutant and wtAAV8 in Balb/c mice using Ang-(1-7) as a target gene. Ang-(1-7) in serum samples was detected at different time points after vector injection. As shown in Figure 3A, the Y703F 
mutant elicits significantly greater Ang-(1-7) expression than wtAAV8 at each time point. The DNA copy number in liver tissue showed similar results (Figure 3B). Liver tissue HE staining showed that the hepatic cell cords arranged orderly and hepatocyte morphology was normal in each group, and no significant inflammatory cell infiltration and fibrosis was found, suggesting that this vector did not cause obvious hepatic toxicity (Figure 3C). These data suggested that the Y703F mutation of AAV8 led to a significant improvement in in vivo gene delivery, primarily in liver tissue. Moreover, none of these vectors caused any obvious liver toxicity.

\section{Ang-(1-7) overexpression induced by the optimized vector inhibits hepatocellular carcinoma growth in vivo.}

The expression levels of MasR were first analyzed in a panel of hepatoma cell lines by real-time RT-PCR. The results demonstrated that levels of MasR were significantly higher in malignant HCC cell lines, including QGY-7701, SMMC-7721, Bel-7402 and H22, compared with highly differentiated HepG2 cells and normal hepatocyte LO2 cells (Figure 4A). This may suggest that MasR serves as the receptor that mediates the anti-proliferative role of Ang-(1-7) in human hepatoma cells. The Y703F mutant leads to

A

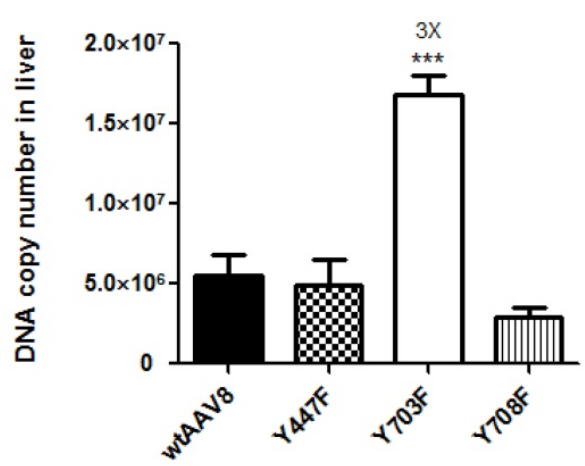

C

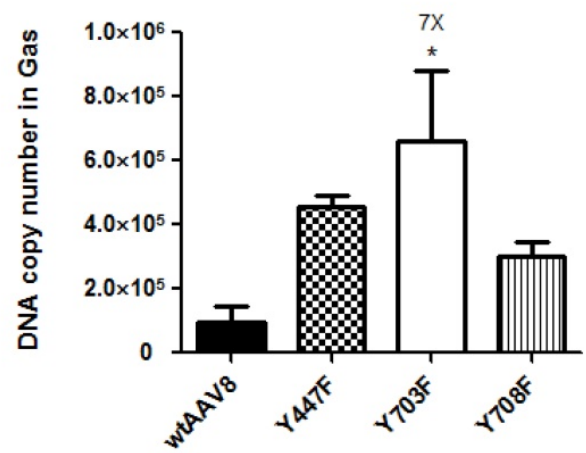

significant enhancement in in vivo gene delivery and specific liver tropism, demonstrating great potential in liver gene therapy applications. Due to the short half-life of Ang-(1-7) peptide, we next explored the anti-tumor effect of Ang-(1-7) overexpression mediated by the optimized vector in $\mathrm{H} 22$ hepatoma-bearing mice. When xenograft tumor volumes had reached $\sim 100 \mathrm{~mm}^{3}$, mice were injected with Y703F-Ang-(1-7), Y703F-Luc or PBS in the same volume via the tail vein. Tumor volumes were measured every 3-5 days for a period of 21 days. The tumor volume in the Y703F-Ang-(1-7)-treated group was significantly smaller than in Y703F-Luc and PBS groups from 5 days after vector injection, while there were no differences between the Y703F-Luc and PBS groups (Figure 4B). At the end of the study, the tumor weight and size in the Y703F-Ang-(1-7) group was markedly reduced compared with the control groups (Figure 4C, 4D), while the Ang-(1-7) relative level in the Y703F-Ang-(1-7) group was significantly higher than in Y703F-Luc and PBS groups, as expected (Figure 4E). These data strongly suggested that Y703F-Ang-(1-7) produced steady and robust Ang-(1-7) expression in vivo, which was associated with significant growth inhibition of hepatocellular carcinoma.

B

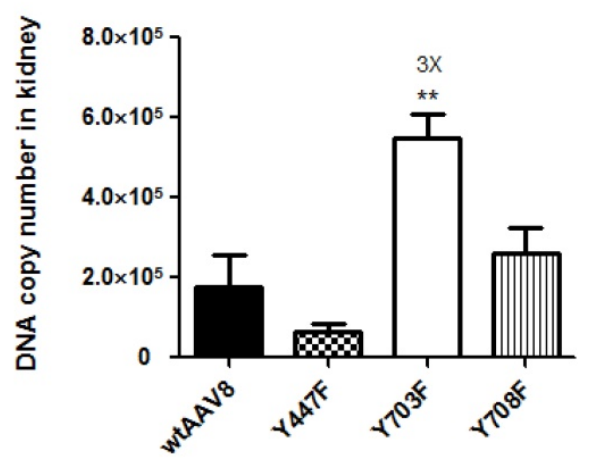

D

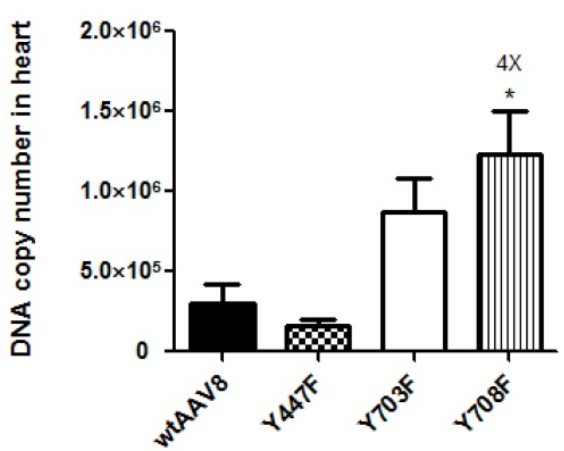

Figure 2. Analysis of viral genome DNA copy numbers in selected tissues After in vivo bioluminescence analysis, animals were killed, genomic DNA was isolated from different tissue samples and $100 \mathrm{ng}$ of each was used in triplicate to determine Firefly luciferase genome copies. The mutant of Y703F showed 3-fold enhancement in liver and kidney tissues and 7-fold higher levels in gastrocnemius (Gas) gene delivery, compared with wild-type AAV8. The data are shown as mean values \pm SD, ${ }^{*} p<0.05$ compared with wild-type AAV8groups. 
A

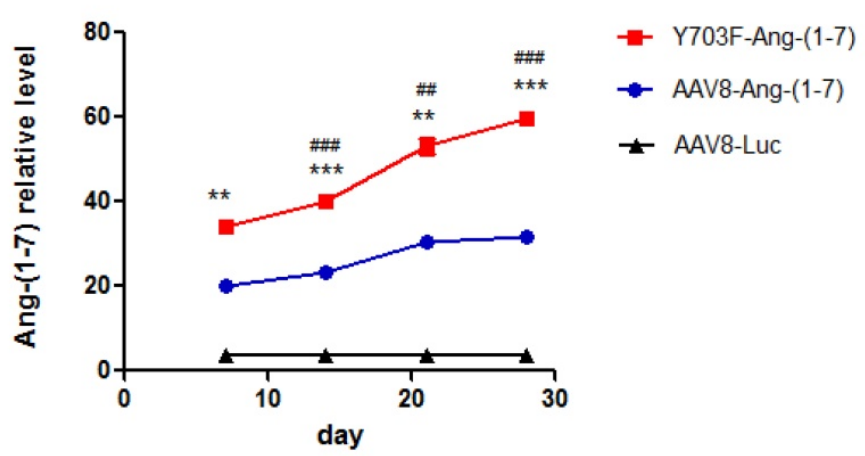

B

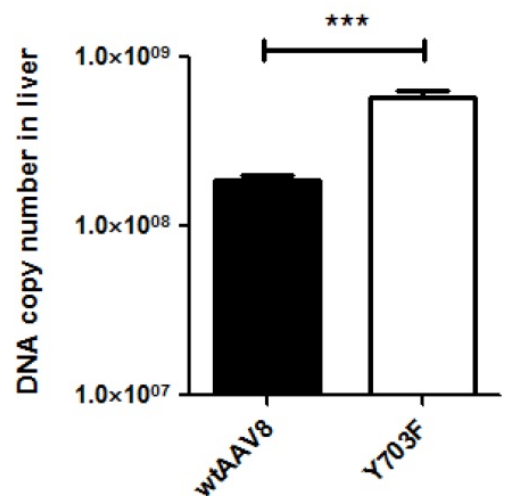

C
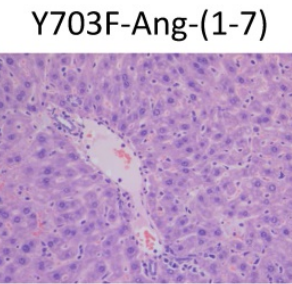

AAV8-Ang(1-7)

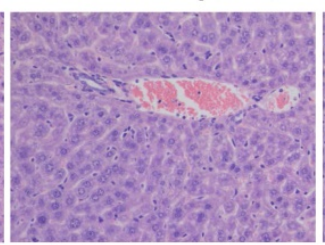

AAV8-Luc

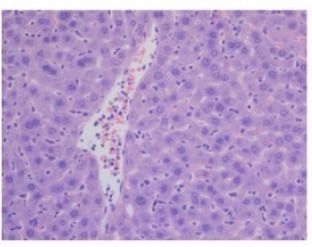

Figure 3. AAV8 and the Y703F mutant vectors mediated sustained and safe expression of Ang-(1-7) in Balb/c mice. $1 \times 1011$ vg of AAV vectors encoding Ang-(1-7) were delivered into 6-8 weeks old Balb/c male mice via tail vein injection. Serum Ang-(1-7) expression levels were detected at 7, 14, 21 and 28 days after vector injection by ELISA (A). Liver gene transduction was determined by real-time QPCR, using primers targeting mouse IgG to indirectly determine Ang-(1-7) overexpression (B). Liver toxicity of vectors were detected by hematoxylin-eosin staining at the end of the experiment (C). The data are shown as mean values \pm SD. ${ }^{*} p<0.05$ compared with AAV8-Luc groups, ${ }^{*} p<0.05$ compared with AAV8-Ang-(1-7) groups.

A

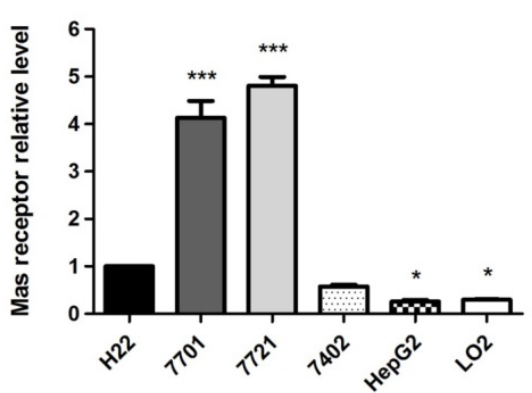

C

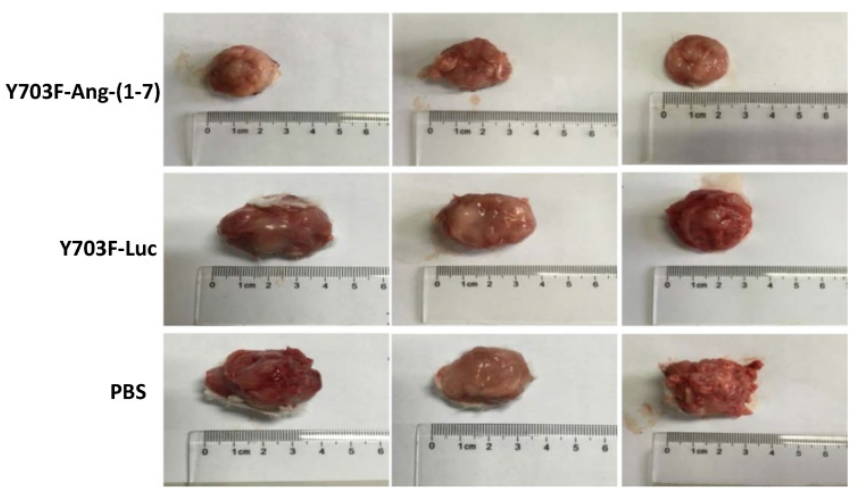

B

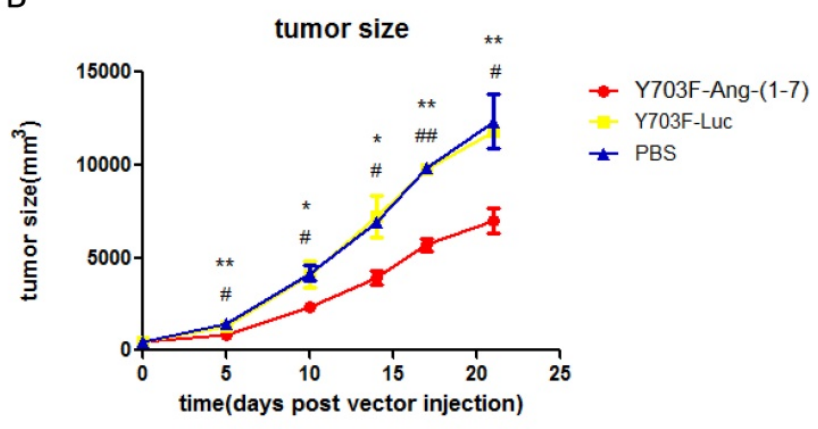

D

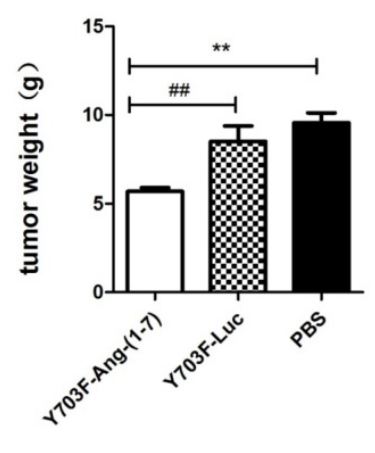

$\mathrm{E}$

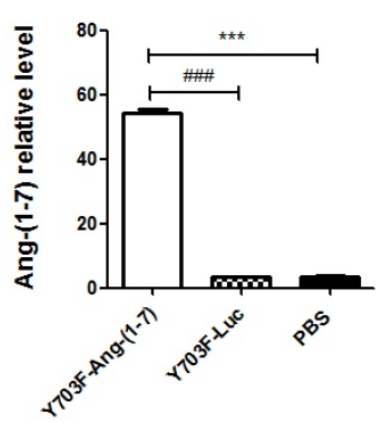

Figure 4. Ang-(1-7) inhibits the growth of hepatocellular carcinoma. The expression level of Mas receptor in hepatoma cell lines was analyzed by real-time RT-PCR. Mas receptor abundance was normalized to GAPDH, The data are shown as mean values \pm SD. * $p<0.05$ compared with $\mathrm{H} 22$ cell line $(\mathrm{A})$. Growth curves of tumor xenografts volumes. Volume $=\left(D \times d^{2}\right) / 2$, where $D$ is the longest diameter and $d$ is the shortest diameter $(B)$; Tumors from mice treated with Y703F-Ang-(1-7), Y703F-Luc or PBS were weighed at the time of sacrifice (C); Size of tumor xenografts from mice injected with Y703F-Ang-(1-7), Y703F-Luc or PBS (D). Levels of Ang-(1-7) in the sera of controls (Y703F-Luc and PBS-treated mice) and Y703F-Ang-(1-7)-treated mice, determined by ELISA (E). The data are shown as mean values \pm SD. ${ }^{*} p<0.05$ compared with PBS groups, ${ }^{*} p<0.05$ compared with $Y 703 F-$ Luc groups. 


\section{Ang-(1-7) overexpression inhibits tumor growth through its anti-angiogenic and anti-proliferative activity}

Ang-(1-7) expression level in liver tissue was measured by immunohistochemical staining using Ang-(1-7) fusion protein (mouse IgG) antibody. The results showed that IgG staining was significantly higher in tumor section from Y703F-Ang-(1-7) treated mice, as compared with other two groups (Figure 5A, 5B). To determine whether Ang-(1-7) overexpression inhibits angiogenesis within tumor xenografts, tumor sections were detected by immunohistochemical staining using VEGF and CD34 antibodies. The tumor tissue sections from Y703F-Ang-(1-7) treated animals exhibited a significant decrease in VEGF expression and CD34-stained vessel density (Figure 5A, 5B). Next, the anti-proliferative activity of Ang-(1-7) was detected using Ki67 and Cdc6 antibodies. Ki67 is a nuclear protein that is strictly associated with cell proliferation. Cdc6 (cell division cycle 6) is an essential regulator of DNA replication in eukaryotic cells. Both are important markers for cell proliferation. Our study showed that a significantly less percentage of Ki67- or Cdc6-stained cells were observed in Y703F-Ang-(1-7) treated animals (Figure 6A, 6B). Liver tissue HE staining showed that the hepatic cell cords arranged orderly and hepatocyte morphology was normal in each group, and no significant inflammatory cell infiltration and fibrosis was found (Figure 6A). These data suggested that Ang-(1-7) overexpression mediated by the optimized AAV vector inhibited tumor cell growth through its anti-angiogenic and anti-proliferative activity, which did not cause any obvious liver toxicity.

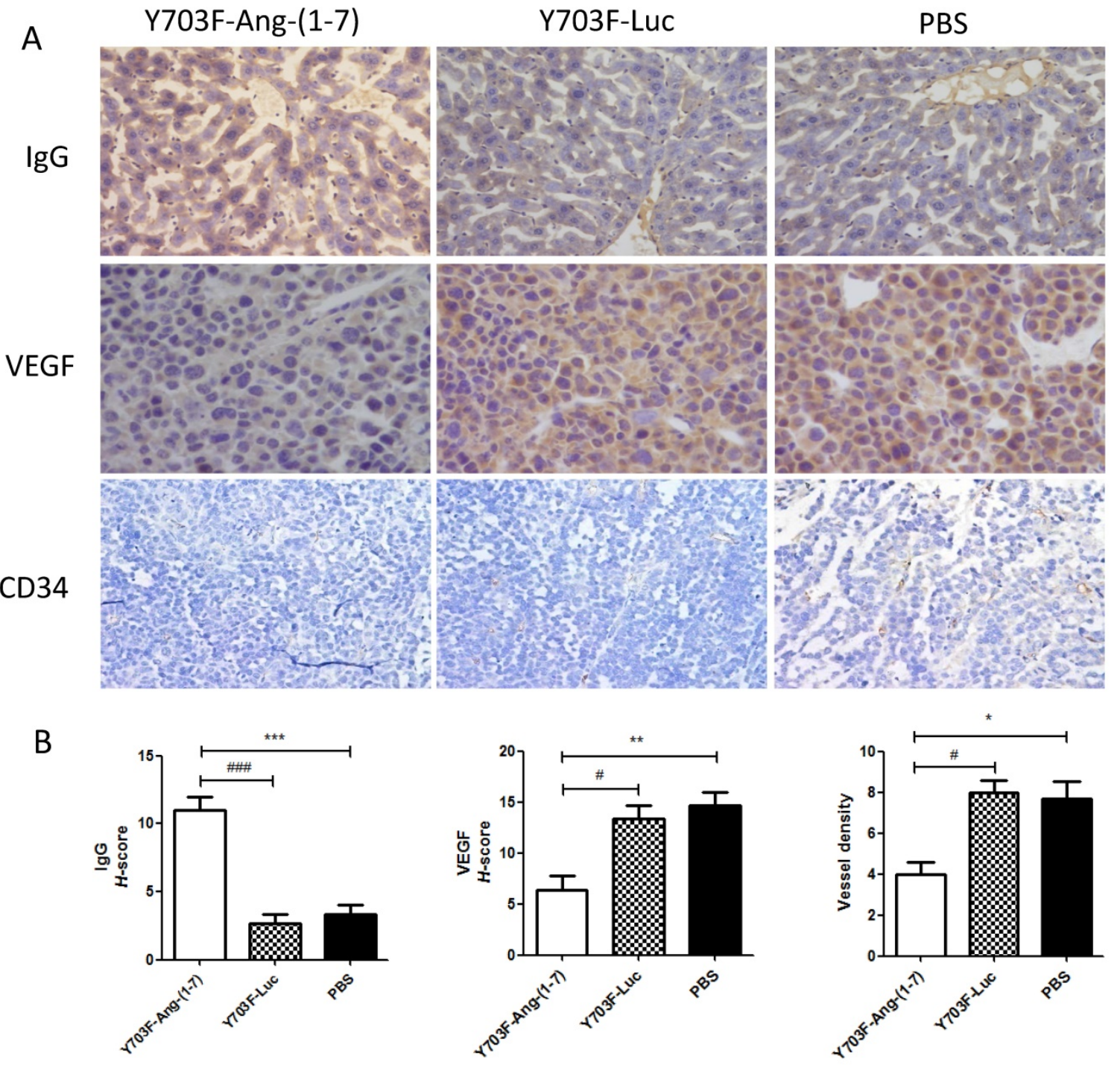

Figure 5. Effect of Ang-(1-7) on angiogenic factors in tumor xenografts. The expression of Ang-(1-7) fusion protein mouse lgG and angiogenic factor VEGF, CD34 in tumor sections from Y703F-Ang-(1-7), Y703F-Luc or PBS-treated mice were determined through immunostaining; Representative photomicrographs are shown at the top (magnification, $\times 400)(A)$; The histochemical score ( $H$-score) was obtained by multiplying the intensity by the percentage of IgG, VEGF or CD34 positive cells (B); bars, SD. * $p<0.05$ compared with PBS groups, ${ }^{*} p<0.05$ compared with Y703F-Luc groups. 

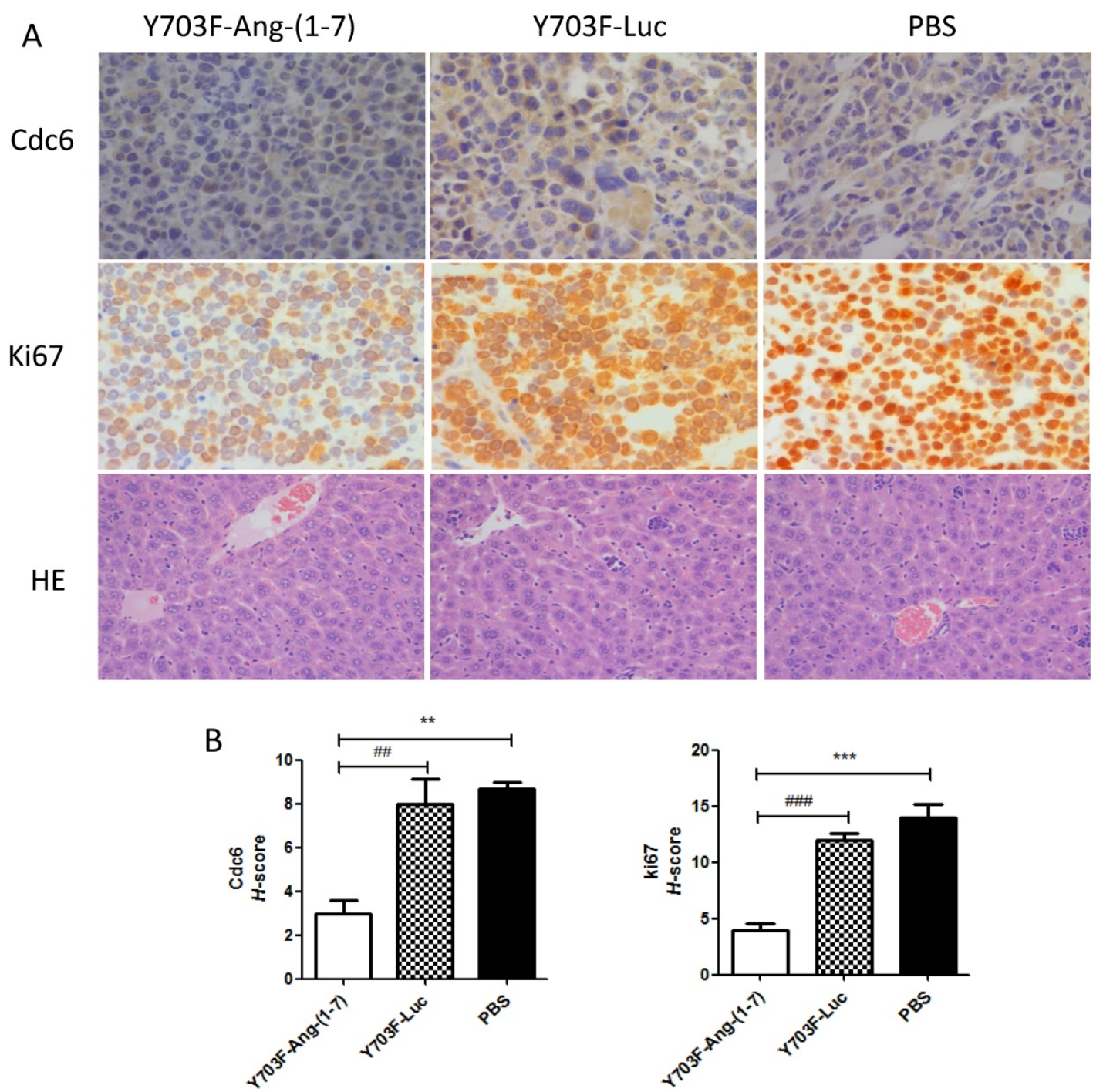

Figure 6. Effect of Ang-(1-7) on proliferative factors in tumor xenografts. Cell proliferative factor Ki67, Cdc6 in tumor sections from Y703F-Ang-(1-7), Y703F-Luc or PBS-treated mice were determined through immunostaining; Liver toxicity of Ang-(1-7) were detected by HE staining. Representative photomicrographs are shown at the top (magnification, $\times 400)(A)$; The histochemical score $(H$-score) was obtained by multiplying the intensity by the percentage of Ki67 or Cdc6 positive cells (B); bars, SD. ${ }^{*} p<0.05$ compared with PBS groups, ${ }^{2} p<0.05$ compared with Y703F-Luc groups.

\section{Effects of Ang-(1-7) overexpression on VEGF, PIGF and VEGF receptors}

The angiogenesis-promoting factor vascular endothelial growth factor (VEGF) and its receptors are known to be necessary for tumor growth and progression. Thus, we further evaluated the effects of Ang-(1-7) overexpression on these angiogenic factors in tumor samples by real-time RT-PCR. As showed in Figure 7, when Ang-(1-7) was overexpressed VEGF and PIGF mRNA levels were reduced by more than $80 \%$ (Figure 7A, 7B), and levels of the VEGF receptors, Flt- 1 and Flk-1, were also shown to be significantly decreased, when compared with control groups (Figure 7C, 7D). These data suggested that Ang-(1-7) overexpression may decrease VEGF and PIGF levels in tumor tissue and attenuate VEGF and PIGF signaling by reducing the number of receptors available to these ligands, so as to inhibit tumor growth.

\section{Discussion}

The clinical potential of Ang-(1-7) as a therapeutic agent to treat hypertension and other cardiovascular diseases is evidenced by the variety of patents and clinical trials involving this peptide [27]. Cancer treatment is one of the most advanced therapeutic areas, and a growing body of evidence suggests that Ang-(1-7) is a promising anti-tumor factor. However, the short half-life severely hampers the in vivo application of this peptide [13]. Adeno-associated virus (AAV) is one of the most versatile vectors for in vivo gene therapy. Our previous study demonstrated that administration of AAV8-Ang-(1-7) significantly attenuated the growth of human nasopharyngeal carcinoma (NPC) xenograft 
tumors and non-small cell lung cancer (NSCLC), suggesting that AAV-mediated Ang-(1-7) gene therapy is potentially effective and useful for cancer treatment $[6,7]$. In this study, we first reported the in vivo anti-tumor efficacy of Ang-(1-7) mediated by the optimized AAV8 in a murine $\mathrm{H} 22$ hepatoma model. We constructed three $\mathrm{Y}$ to $\mathrm{F}$ mutants of AAV8 and evaluated their transduction efficiency in C57BL/6 mice. As shown in the results (Figure 2), the Y703F mutant that we constructed induced 3- to 7- fold higher gene expression than wtAAV8 in liver, kidney, and gastrocnemius gene delivery, implying its utility in transduction of those tissues. We next explored the in vivo effects of Ang-(1-7) mediated by the Y703F mutant. Our data demonstrated that the optimized AAV vector mediated long-term and high efficiency expression of Ang-(1-7) in hepatoma-bearing Balb/c mice, which resulted in significant inhibition of tumor growth and tumor angiogenesis. Our study suggested that AAV-based gene delivery is a potentially effective method for Ang-(1-7) in hepatocellular carcinoma (HCC) therapy.

The anti-proliferative and anti-angiogenic effects of Ang-(1-7) have been verified in a variety of cancer cells, including lung cancer, prostate cancer, breast cancer, and nasopharyngeal carcinoma [28]. In this

A

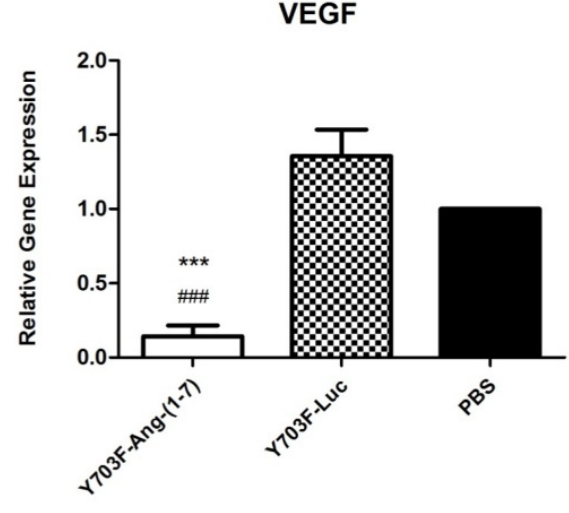

C

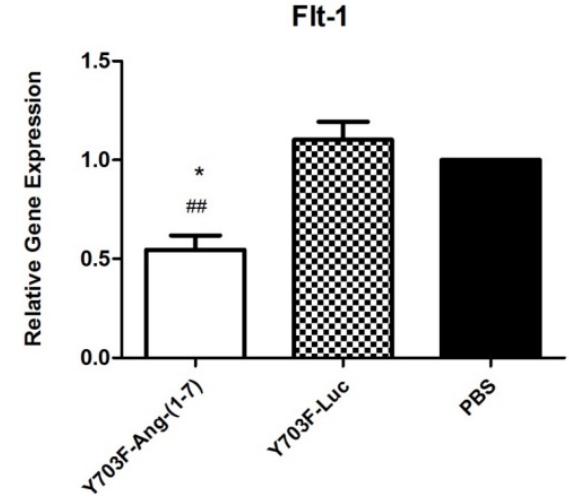

study, we demonstrated that Ang-(1-7) overexpresssion significantly inhibits the growth of hepatocellular carcinoma, with a remarkable reduction in tumor size and tumor weight (Figure 4). Importantly, we also detected significant decreases in the levels of PIGF, VEGF, VEGF receptor 1 (Flt-1) and VEGF receptor 2 (Flk-1) mRNAs in the tumors of mice treated with Ang-(1-7), when compared with tumors from control animals (Fig. 7). The angiogenesis-promoting factors, VEGF and PIGF, play an important role in tumor growth and progression. Thus these data suggested that Ang-(1-7) may exert anti-HCC proliferation effect by decreasing the expression of the proangiogenic genes PIGF, VEGF and their receptors. Cell division cycle 6 (Cdc6) is essential for DNA replication and its deregulation is involved in carcinogenesis. High levels of CDC6 have been recently reported in around $50 \%$ of NSCLC, brain cancer and a subset of mantle cell lymphomas, which suggests that Cdc6 has oncogenic properties. Our previous study demonstrated that Ang-(1-7) over-expression can markedly suppress NSCLC growth in vivo by down-regulating Cdc6. In this study we found that Cdc6 expression level was significantly reduced in Y703F-Ang-(1-7) treated group (Figure 6A, 6B), which further verified the anti-Cdc6 effect of Ang-(1-7).

B

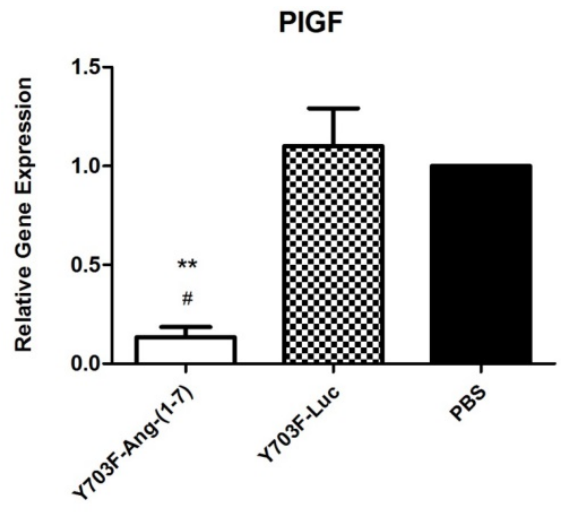

D

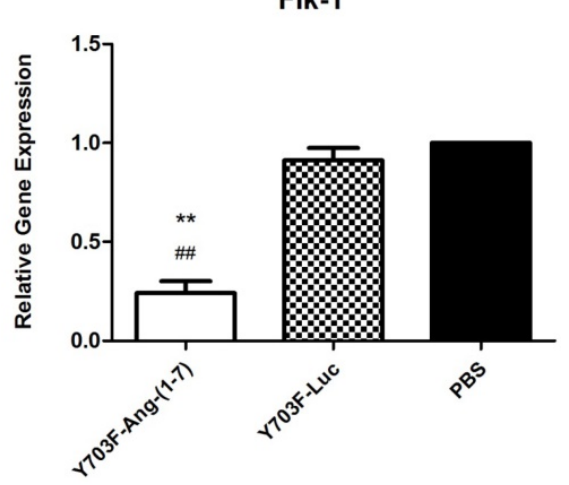

Figure 7. Ang-(1-7) inhibited the expression of angiogenic factors PIGF, VEGF and VEGF receptors in tumor samples. The expression level of PIGF, VEGF and VEGF receptors between Y703F-Ang-(1-7), Y703F-Luc or PBS groups were determined by real-time RT-PCR, and data were quantified by the comparative $\triangle \triangle \mathrm{Ct}$ method by using human GAPDH as the internal standard. ${ }^{*} p<0.05$ compared with PBS groups, \# $p<0.05$ compared with $Y 703 \mathrm{~F}-$ Luc groups. 
One of the biggest challenges hindering Ang-(1-7)'s therapeutic use is degradation, as it is a seven-amino acid peptide and the in vivo half-life time is very short. Several solutions are mentioned in patent documents such as cyclization, modification or extension of amino acids, and combination with other compounds [28]. In other studies mice have been treated with Ang-(1-7) via continuous infusion from osmotic minipumps [29]. Our previous study demonstrated that AAV-based gene therapy is potentially effective and useful for Ang-(1-7) delivery in cancer gene therapy. Building on this, we attempted to minimize the vector producing cost and intracellular vector degradation to further improve AAV in vivo transduction.

It has been widely reported that tyrosine mutations in AAV8 can mediate enhanced gene delivery in liver, retina and lung tissues [22, 30, 31]. In this study, we have successfully generated three tyrosine mutants of the AAV8 capsid with differing and tissue specific gene delivery enhancements. However, none of the constructs made in the current study could elicit 10- to 20-fold enhancement such as those observed in the retina from previous findings [21]. Notably, tyrosine phosphorylation may play an important role in these tissues. Certain mutant vectors can successfully escape phosphorylation and proteasome-mediated degradation and thus achieve more efficient gene delivery. The route of administration may also be an important variable in our experiments. In our study, vectors were delivered by tail vein injection, which results in wider vector distribution than subretinal or intravitreal injection. However, the consequence might be that tail vein injected vectors are more susceptible to the humoral immune response leading to vector degradation. Lastly, mutations on the AAV capsid may also affect its interaction with cellular receptors, intracellular trafficking to the nucleus, and host immune recognition during the infection [32]. Our study demonstrated that the mutant Y703F elicited a significant enhancement in systemic gene expression level and liver tropism (Figures 1,2), suggesting great potential in improving gene therapy applications.

\section{Conclusions}

In summary, the AAV8 capsid mutant (Y703F) generated in this study led to significant improvements in systemic transgene expression and long-term and high efficiency expression of Ang-(1-7) in vivo, resulting in significant attenuation of the growth of hepatocellular carcinoma by decreasing expression of the proangiogenic and proliferative factors. These data suggested that AAV8 (Y703F)-mediated Ang-(1-7) may serve as a useful and efficient approach for hepatocellular carcinoma gene therapy.

\section{Acknowledgements}

The authors acknowledge Shengyao Wang and Qingqing Li for their technical assistance and help with real time RT-PCR.

\section{Funding}

This study was financially supported by the Industrial High-tech Fields of Science and Technology Plan Projects of Guangdong Province grant 2013B010404026, the National High Technology Research and Development Program of China 2015AA020946 (863 Program), Beijing Nova Program Z171100001117119, the 61-class General Financial Grant from the China Postdoctoral Science Foundation 2017M612696.

\section{Authors' contributions}

Conception and design: YM, NP, XC, XW, SW, HL. Development of methodology: YM, NP, XW, CS, SW, HL. Acquisition of data (provided animals, provided facilities, etc.): XC, HC, RY, NB. Analysis and interpretation of data (e.g., statistical analysis, biostatistics, computational analysis): YM, NP, XW, JL, YZ, SW, HL. Writing, review, and/or revision of the manuscript: YM, NP, XW, AL, CS, SW, HL. Administrative, technical, or material support (i.e., reporting or organizing data, constructing databases): YM, NP, XW, HD, BC. Study supervision: SW, HL. All authors read and approved the final manuscript.

\section{Competing Interests}

The authors have declared that no competing interest exists.

\section{References}

1. Lafaro KJ, Demirjian AN, Pawlik TM. Epidemiology of hepatocellular carcinoma. Surgical oncology clinics of North America. 2015; 24: 1-17.

2. Yang JD, Roberts LR. Hepatocellular carcinoma: A global view. Nat Rev Gastroenterol Hepatol. 2010; 7: 448-58.

3. Tang X, Huang J, Xiong H, Zhang K, Chen C, Wei X, et al. Anti-Tumor Effects of the Polysaccharide Isolated from Tarphochlamys Affinis in $\mathrm{H} 22$ Tumor-Bearing Mice. Cellular physiology and biochemistry: international journal of experimental cellular physiology, biochemistry, and pharmacology. 2016; 39: 1040-50.

4. Kota J, Chivukula RR, O'Donnell KA, Wentzel EA, Montgomery CL, Hwang $\mathrm{HW}$, et al. Therapeutic microRNA delivery suppresses tumorigenesis in a murine liver cancer model. Cell. 2009; 137: 1005-17.

5. Li H, Qi Y, Li C, Braseth LN, Gao Y, Shabashvili AE, et al. Angiotensin type 2 receptor-mediated apoptosis of human prostate cancer cells. Molecular cancer therapeutics. 2009; 8: 3255-65.

6. Pei N, Wan R, Chen X, Li A, Zhang Y, Li J, et al. Angiotensin-(1-7) Decreases Cell Growth and Angiogenesis of Human Nasopharyngeal Carcinoma Xenografts. Molecular cancer therapeutics. 2016; 15: 37-47.

7. Chen X, Chen S, Pei N, Mao Y, Wang S, Yan R, et al. AAV-Mediated angiotensin 1-7 overexpression inhibits tumor growth of lung cancer in vitro and in vivo. Oncotarget. 2017; 8: 354-63.

8. Tallant EA. Angiotensin-(1-7) inhibits growth of cardiac myocytes through activation of the mas receptor. AJP: Heart and Circulatory Physiology. 2005; 289: H1560-H6.

9. Wegman-Ostrosky T, Soto-Reyes E, Vidal-Millan S, Sanchez-Corona J. The renin-angiotensin system meets the hallmarks of cancer. Journal of the renin-angiotensin-aldosterone system: JRAAS. 2015; 16: 227-33. 
10. Rodgers KE, Oliver J, diZerega GS. Phase I/II dose escalation study of angiotensin 1-7 [A(1-7)] administered before and after chemotherapy in patients with newly diagnosed breast cancer. Cancer chemotherapy and pharmacology. 2006; 57: 559-68.

11. Petty WJ, Miller AA, McCoy TP, Gallagher PE, Tallant EA, Torti FM. Phase I and pharmacokinetic study of angiotensin-(1-7), an endogenous antiangiogenic hormone. Clin Cancer Res. 2009; 15: 7398-404.

12. Savage PD, Lovato J, Brosnihan KB, Miller AA, Petty WJ. Phase II Trial of Angiotensin-(1-7) for the Treatment of Patients with Metastatic Sarcoma. Sarcoma. 2016; 2016: 4592768.

13. Qi Y, Shenoy V, Wong F, Li H, Afzal A, Mocco J, et al. Lentivirus-mediated overexpression of angiotensin-(1-7) attenuated ischaemia-induced cardiac pathophysiology. Experimental physiology. 2011; 96: 863-74.

14. Carpentier AC, Frisch F, Labbe SM, Gagnon R, de Wal J, Greentree S, et al. Effect of alipogene tiparvovec (AAV1-LPL(S447X)) on postprandial chylomicron metabolism in lipoprotein lipase-deficient patients. The Journal of clinical endocrinology and metabolism. 2012; 97: 1635-44.

15. Testa F, Maguire AM, Rossi S, Pierce EA, Melillo P, Marshall K, et al. Three-year follow-up after unilateral subretinal delivery of adeno-associated virus in patients with Leber congenital Amaurosis type 2. Ophthalmology. 2013; 120: 1283-91.

16. Nathwani AC, Tuddenham EG, Rangarajan S, Rosales C, McIntosh J, Linch $\mathrm{DC}$, et al. Adenovirus-associated virus vector-mediated gene transfer in hemophilia B. N Engl J Med. 2011; 365: 2357-65.

17. Mak KY, Rajapaksha IG, Angus PW, Herath CB. The Adeno-Associated Virus - A Safe and Effective Vehicle for Liver-Specific Gene Therapy of Inherited and Non-Inherited Diseases. Current gene therapy. 2017.

18. Chen $\mathrm{Q}$, Zhai $\mathrm{H}, \mathrm{Li} \mathrm{X}, \mathrm{Ma} \mathrm{Y}$, Chen B, Liu F, et al. Recombinant adeno-associated virus serotype 9 in a mouse model of atherosclerosis: Determination of the optimal expression time in vivo. Molecular medicine reports. 2017.

19. Cabral-Miranda F, Nicoloso-Simoes E, Adao-Novaes J, Chiodo V, Hauswirth WW, Linden R, et al. rAAV8-733-Mediated Gene Transfer of CHIP/Stub-1 Prevents Hippocampal Neuronal Death in Experimental Brain Ischemia. Molecular therapy: the journal of the American Society of Gene Therapy. 2017; 25: 392-400.

20. Qiu Y, Tao L, Zheng S, Lin R, Fu X, Chen Z, et al. AAV8-Mediated Angiotensin-Converting Enzyme 2 Gene Delivery Prevents Experimental Autoimmune Uveitis by Regulating MAPK, NF-kappaB and STAT3 Pathways. Scientific reports. 2016; 6: 31912.

21. Petrs-Silva $\mathrm{H}$, Dinculescu A, Li Q, Min SH, Chiodo V, Pang JJ, et al High-efficiency transduction of the mouse retina by tyrosine-mutant AAV serotype vectors. Molecular therapy: the journal of the American Society of Gene Therapy. 2009; 17: 463-71.

22. Deng WT, Dinculescu A, Li Q, Boye SL, Li J, Gorbatyuk MS, et al. Tyrosine-mutant AAV8 delivery of human MERTK provides long-term retinal preservation in RCS rats. Invest Ophthalmol Vis Sci. 2012; 53: 1895-904.

23. Gabriel N, Hareendran S, Sen D, Gadkari RA, Sudha G, Selot $R$, et al. Bioengineering of AAV2 capsid at specific serine, threonine, or lysine residues improves its transduction efficiency in vitro and in vivo. Human gene therapy methods. 2013; 24: 80-93.

24. Sen D, Gadkari RA, Sudha G, Gabriel N, Kumar YS, Selot R, et al. Targeted modifications in adeno-associated virus serotype 8 capsid improves its hepatic gene transfer efficiency in vivo. Human gene therapy methods. 2013; 24: $104-16$.

25. Ayuso E, Mingozzi F, Bosch F. Production, purification and characterization of adeno-associated vectors. Current gene therapy. 2010; 10: 423-36.

26. McCarty KS Jr ML, Cox EB, Konrath J, McCarty KS Sr. Estrogen receptor analyses. Correlation of biochemical and immunohistochemical methods using monoclonal antireceptor antibodies. Arch Pathol Lab Med 1985; 109: 716-21.

27. Santos RA. Angiotensin-(1-7). Hypertension. 2014; 63: 1138-47.

28. Machado-Silva A, Passos-Silva D, Santos RA, Sinisterra RD. Therapeutic uses for Angiotensin-(1-7). Expert opinion on therapeutic patents. 2016; 26: 669-78.

29. Cook KL, Metheny-Barlow LJ, Tallant EA, Gallagher PE. Angiotensin-(1-7) reduces fibrosis in orthotopic breast tumors. Cancer research. 2010; 70: 8319-28.

30. Martini SV, da Silva AL, Ferreira D, Gomes K, Ornellas FM, Lopes-Pacheco M, et al. Single tyrosine mutation in AAV8 vector capsid enhances gene lung delivery and does not alter lung morphofunction in mice. Cellular physiology and biochemistry: international journal of experimental cellular physiology, biochemistry, and pharmacology. 2014; 34: 681-90.

31. Qi YF, Li QH, Shenoy V, Zingler M, Jun JY, Verma A, et al. Comparison of the transduction efficiency of tyrosine-mutant adeno-associated virus serotype vectors in kidney. Clinical and experimental pharmacology \& physiology. 2013; 40: 53-5.

32. Petrs-Silva H, Dinculescu A, Li Q, Deng WT, Pang JJ, Min SH, et al. Novel properties of tyrosine-mutant AAV2 vectors in the mouse retina. Molecular therapy: the journal of the American Society of Gene Therapy. 2011; 19: 293-301. 